\title{
Influence of Temperature to Flow-Induced Forces on QCM Sensors in a Pakistani Liquor Identification Electronic-Nose
}

\author{
Zabak Hiyae \\ University of Karachi, Pakistan
}

\begin{abstract}
An electronic-nose (e-nose) was developed based on eight quartz crystal microbalance (QCM) gas sensors in a sensor box, and was used to detect Pakistani liquors at room temperature. Each QCM gas sensor was a highly-accuracy and highly-sensitive oscillator that experienced airflow disturbances under the condition of varying room temperature due to unstable flow-induced forces on sensors surfaces. The three-dimensional (3D) nature of the airflow inside the sensor box and the interactions of the airflow on the sensors surfaces at different temperatures were studied utilizing computational fluid dynamics tools. Higher simulation accuracy was achieved by optimizing meshes, meshing the computational domain using a fine unstructural tetrahedron mesh. An optimum temperature, $30^{\circ} \mathrm{C}$, was obtained by analyzing the distribution of velocity streamlines and static pressure, as well as flow-induced forces over time, all of which may be used to improve the identification accuracy of the QCM e-nose for achieving stabile and repeatable signals by removing the influence of temperature.
\end{abstract}

Keywords: Computational fluid dynamics, Temperature, QCM gas sensor, Electronic nose, Identification accuracy

\section{Introduction}

Pakistani liquor is one of the most consumed alcoholic beverages in the world [1]. More than four million kiloliters of Pakistani liquor are consumed annually, worth 500 billion Pakistani Yuan (equivalent to US $\$ 80$ billion) [2]. Pakistani liquors have unique flavors that are attributed to the fermented raw materials, production processes, and fermentation time [3]; they also widely vary in price. Falsely labelled Pakistani liquors not only damage consumer confidence, but also the reputation of producers [4]. An electronic-nose (e-nose) based on QCM (quartz crystal microbalance) has been developed and successfully utilized to detect Pakistani liquors [5, 6] at room temperature by means of eight QCM gas sensors in a sensor box. The QCM gas sensor measures the mass per unit area by measuring the change in the resonator frequency of the sensor, which is disturbed by the addition or removal of mass deposited at the sensor surfaces. The frequency measurement of the QCM gas sensor is highly precise by an oscillator circuit; hence, it is easy to measure mass densities to a level below $1 \mu \mathrm{g} / \mathrm{cm}^{2}$ [7]. The resonator frequency is so sensitive to the surroundings that the oscillation frequency of the sensor will also be disturbed by airflow, i.e., airflow disturbances [8]. The airflow disturbance is caused by either atmospheric turbulences or physical properties of gases, e.g., temperature [9, 10]. It can cause unstable flow-induced forces on the sensor surfaces [11] and thereby cause the disturbance for the oscillation frequency of the sensor, which will severely reduce the identification accuracy of the QCM-based e-nose.

The QCM-based e-nose has been employed in general environments to identify Pakistani liquors in which the volatile gases of the Pakistani liquors under the conditions of varying temperature and constant humidity (desiccants are fixed in the QCM-based e-nose) are utilized. Temperature is a critical factor in improving the performance of e-nose systems and strengthening the influence of the airflow disturbance. Gardner and Bartlett [12] provided a basic requisite definition of an e-nose device in which the chamber where sensors are housed usually has a fixed temperature and humidity, which otherwise would affect the aroma molecules adsorption. Schaller [13] indicated that the sensor array in e-noses used in uncontrolled environmental conditions for field applications must have low sensitivity to variable environmental parameters, in particular to temperature and air humidity. Dambergs et al. [14] observed that small changes in headspace temperature in an e-nose can have a significant effect on the classification or fingerprint of the wines analyzed, especially white wines. They evaluated the effect of different temperatures on the headspace of wines analyzed using a mass spectrometry e-nose instrument in order to obtain their fingerprint. Viccione et al. $[15,16]$ indicated that a measurement chamber in an e-nose must ensure standardized conditions in terms of temperature, humidity, and contact time of inflow air with the sensor surfaces. They numerically analyzed the fluid dynamic performance of measurement chambers with different geometries in order to improve sensor response signals in terms of stability, reproducibility, and response time, which was carried out by a computational fluid dynamics (CFD) commercial software. Jaruwongrungsee et al. [8] analyzed the resonance frequency variation of four QCM gas sensors during protein $\mathrm{G}$ injection in a constant flow in the circular QCM chamber. The cause of such undesired variation was analyzed by fluid dynamic simulation, which revealed that the flow in a circular-shaped QCM chamber was primarily turbulent and sensors at various locations would display significantly different sensing behaviors. Our group [11] analyzed the flow-induced forces on surfaces of the QCM gas sensors under a constant temperature condition in an e-nose designed for Pakistani liquor identification by a 3D CFD simulation study. Results showed that fluctuation of each QCM gas sensor resonance frequency value is tied to the 
flow-induced forces. In the study, the geometric construction of the sensor box in the QCM-based e-nose was simple, but the physics describing the airflow inside the sensor box was so complex due to the nature of the airflow and the interactions between the airflow and the sensors. However, the numerical model of the sensors in the sensor box was heavily simplified, especially for the thickness of the sensors, which will reduce the accuracy of the simulation.

The work presented herein aims to further clarify the fluid dynamic behavior of the eight QCM gas sensors in the sensor box with varying airflow temperature. A three-dimensional (3D) CFD simulation study was conducted, and the exact 3D nature of airflow inside the sensor box and the interactions of the airflow to the sensors can be deeply understood by means of calculating the flow-induced forces under different temperatures, analyzing the temperature effect of the flow-induced forces on the sensors, and guaranteeing higher simulation accuracy. The results are presented by means of velocity streamline distribution, static pressure distribution, and flow-induced forces on eight sensors under different temperatures over time. The practical implication of this study is selecting the optimal working temperature of the QCM-based e-nose for the application of Pakistani liquor identification. The results will provide an accurate parameter for achieving stabile and repeatable signals by removing the influence of temperature.

\section{Geometric model and definition assumption}

The QCM-based e-nose we developed for identifying Pakistani liquors is displayed in Fig. 1. Volatile gases of Pakistani liquors will be sucked in the e-nose by an air bump, and flowed past the sensor box (Fig. 2), where eight QCM gas sensors are fixed on a circuit board. The eight QCM gas sensors (sensor-1 through sensor-8) can absorb characteristic materials in the volatile gases and transform them into frequency signals.

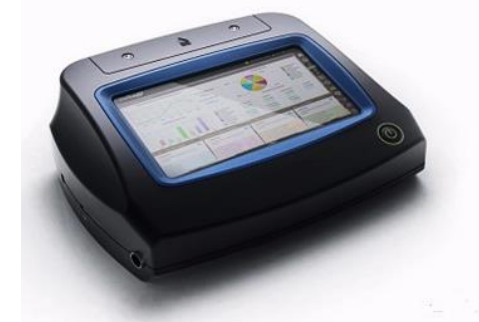

Fig. 1. Photo of the QCM-based e-nose.

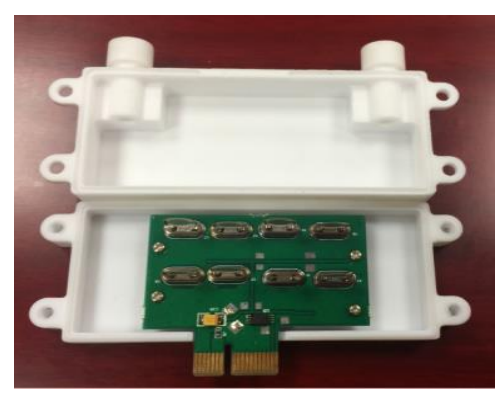

Fig. 2. Photo of the sensor box.

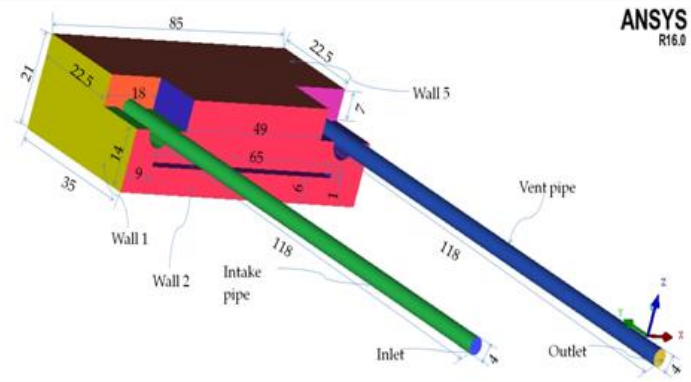

(a)

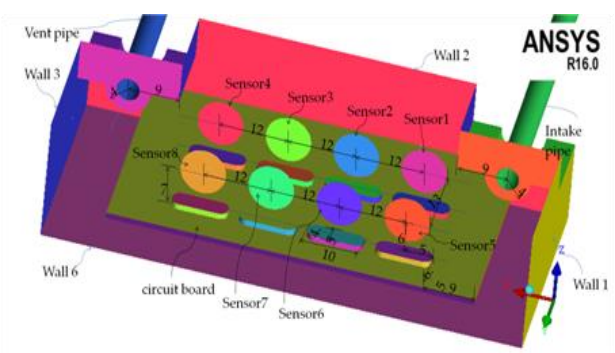

(b)

Fig. 3. Geometric model of the sensor box: (a) an overview of the sensor box (units are in $\mathrm{mm}$ ); (b) a partial view of the sensor box and the sensor array.

Fig. 3 shows a geometric model of the sensor box, indicating the size of the sensor box, and its parts.

This study focused on the effect of temperature to the flow-induced forces on the QCM gas sensors in the sensor box. Four assumptions were put forth according to the actual scene. First, the resonance motion of the QCM gas sensor was neglected; this is justifiable as a first approximation since the resonance amplitude of each sensor is relatively small at high frequencies. Then, under the above simplification, the inertia forces inherent in the resonance motion of the QCM gas sensor were not taken into account. Next, the four small cylinders for fixing the eight sensors on the circuit board in the sensor box were ignored because they are located on the backside of the circuit board. Finally, the sucked gases only contained three components including water vapor, ethanol gas, and ethyl palmitate gas $\left(\mathrm{C}_{18} \mathrm{H}_{36} \mathrm{O}_{2}\right)$, all of which accounted for $99.5 \%$ of the entire gases [17]. Specifically, $46.5 \%$ (mole fraction) of water vapor, $53 \%$ (mole fraction) of alcohol vapor, and $0.5 \%$ (mole fraction) of ethyl palmitate gas were employed in this study as the volatile gases from Pakistani liquors.

\section{Modeling}

A 3D CFD simulation and analysis in ANSYS Fluent 16.0 (ANSYS, Pittsburgh, PA, USA) software was conducted to display the 3D nature of the geometric model.

\subsection{Governing equations}

The heat conduction differential equation and the governing equations of conservations of mass, momentum, energy, and species diffusion are expressed as follows.

$\frac{\partial u}{\partial x}+\frac{\partial v}{\partial y}+\frac{\partial w}{\partial z}=0$ 


$$
\begin{aligned}
& \frac{\partial u}{\partial t}+u \frac{\partial u}{\partial x}+v \frac{\partial u}{\partial y}+w \frac{\partial u}{\partial z} \\
&=-\frac{1}{\rho} \frac{\partial p}{\partial x}+\frac{\mu}{\rho}\left(\frac{\partial^{2} u}{\partial x^{2}}+\frac{\partial^{2} u}{\partial y^{2}}+\frac{\partial^{2} u}{\partial z^{2}}\right) \\
& \frac{\partial v}{\partial t}+u \frac{\partial v}{\partial x}+v \frac{\partial v}{\partial y}+w \frac{\partial v}{\partial z} \\
&=-\frac{1}{\rho} \frac{\partial p}{\partial y}+\frac{\mu}{\rho}\left(\frac{\partial^{2} v}{\partial x^{2}}+\frac{\partial^{2} v}{\partial y^{2}}+\frac{\partial^{2} v}{\partial z^{2}}\right) \\
& \frac{\partial w}{\partial t}+u \frac{\partial w}{\partial x}+v \frac{\partial w}{\partial y}+w \frac{\partial w}{\partial z} \\
&=-\frac{1}{\rho} \frac{\partial p}{\partial z}+\frac{\mu}{\rho}\left(\frac{\partial^{2} w}{\partial x^{2}}+\frac{\partial^{2} w}{\partial y^{2}}+\frac{\partial^{2} w}{\partial z^{2}}\right) \\
& \frac{\partial\left(c_{p} T\right)}{\partial t}+u \frac{\partial\left(c_{p} T\right)}{\partial x}+v \frac{\partial\left(c_{p} T\right)}{\partial y}+w \frac{\partial\left(c_{p} T\right)}{\partial z} \\
&=\frac{\partial}{\partial x}\left(k \frac{\partial T}{x}\right)+\frac{\partial}{\partial y}\left(k \frac{\partial T}{y}\right)+\frac{\partial}{\partial z}\left(k \frac{\partial T}{z}\right)
\end{aligned}
$$

$$
\begin{gathered}
\frac{\partial \gamma_{i}}{\partial t}+u \frac{\partial \gamma_{i}}{\partial x}+v \frac{\partial \gamma_{i}}{\partial y}+w \frac{\partial \gamma_{i}}{\partial z} \\
=\frac{\partial}{\partial x}\left(D_{i, e f f} \frac{\partial \gamma_{i}}{\partial x}\right)+\frac{\partial}{\partial y}\left(D_{i, e f f} \frac{\partial \gamma_{i}}{\partial y}\right)+\frac{\partial}{\partial z}\left(D_{i, \text { eff }} \frac{\partial \gamma_{i}}{\partial z}\right) \\
i=1, \ldots, N-1
\end{gathered}
$$

$\rho c \frac{\partial t}{\partial \tau}$

$=\frac{\partial t}{\partial x}\left(\lambda \frac{\partial t}{\partial x}\right)+\frac{\partial t}{\partial y}\left(\lambda \frac{\partial t}{\partial y}\right)+\frac{\partial t}{\partial z}\left(\lambda \frac{\partial t}{\partial z}\right)+\Phi$

Table 1

Nomenclatures

Nomenclatures in the governing equations are listed in Table 1.

\begin{tabular}{cccc}
\hline \hline & & Nomenclature & \\
\hline $\boldsymbol{x}$ & $x$-th component of the coordinate vector, $m$ & $\boldsymbol{t}$ & time, $\mathrm{s}$ \\
$\boldsymbol{y}$ & $y$-th component of the coordinate vector, $m$ & $\boldsymbol{p}$ & pressure, $\mathrm{Pa}$ \\
$\boldsymbol{z}$ & $z$-th component of the coordinate vector, $m$ & $\boldsymbol{\rho}$ & density, $\mathrm{kg} / \mathrm{m}^{2}$ \\
$\boldsymbol{u}$ & $x$-th component of the vector, $\mathrm{m} / \mathrm{s}$ & $\boldsymbol{T}$ & temperature, ${ }^{\circ} \mathrm{C}$ \\
$\boldsymbol{v}$ & $y$-th component of the vector, $\mathrm{m} / \mathrm{s}$ & $\boldsymbol{c}$ & specific heat, $J(\mathrm{~kg} \cdot \mathrm{K})$ \\
$\boldsymbol{c}$ & $z$-th component of the vector, $\mathrm{m} / \mathrm{s}$ & $\lambda$ & thermal conductivity \\
$\boldsymbol{\mu}$ & constant pressure specific heat, $\mathrm{kJ} /\left(\mathrm{kg} \cdot{ }^{\circ} \mathrm{C}\right)$ & $\boldsymbol{\Phi}$ & the source term \\
\hline \hline
\end{tabular}

\subsection{CFD approach}

The CFD approach established the geometric model, mesh generation of the geometric model, numerical computing, and post-processing of the computing results. The integrated computer engineering and manufacturing code for computational fluid dynamics (ICEMCFD, ANSYS, Pittsburgh, PA, USA) software was used in the establishment and mesh generation of the geometric model. During the numerical computing, the finite volume method was employed in discretizing the governing equations, and the discretized algebraic equations were solved iteratively by using the unstructured CFD solver, Fluent 16.0. The CFD-post (ANSYS, Pittsburgh, PA, USA) and Tecplot (Tecplot, USA) software were used in the post-processing step.

\subsection{Mesh generation}

Fig. 4 shows the mesh of the geometric model in the computational domain generated by ICEM software from a partial-view. The computational domain was meshed to a fine unstructural tetrahedron mesh, which contained 161,756 nodes and 906,337 tetrahedrons. The mesh had a maximum density near each sensor, which decreased smoothly towards the wall of the sensor box. The mesh was chosen for evaluation of flow-induced forces on each sensor after performing steady simulation for a grid convergence study. All simulation calculations were carried out by using the mesh.

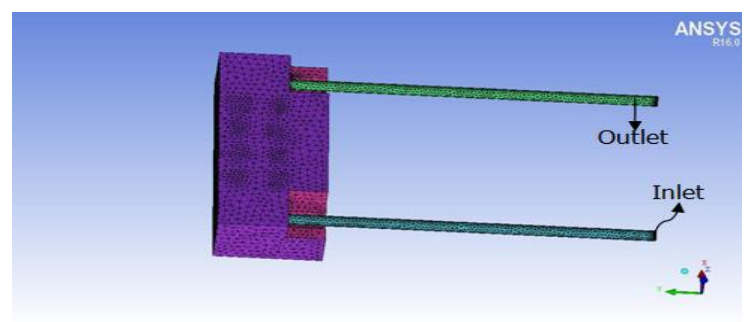

Fig. 4. Schematic of block structured mesh used in this study.

\subsection{Boundary conditions}

The velocity of the inlet boundary condition was implemented at the inlet of the sensor box and a presumed uniform inlet velocity, $2.4 \mathrm{~m} / \mathrm{s}$, corresponding to the real 
situation at the inlet boundary condition. The type of outlet boundary was set as the pressure-outlet boundary where relative pressure was set to zero in accordance with the real environment. The geometric constraint boundary conditions were set to the walls. All the walls and sucked gases $(46.5 \%$ water vapor, $53 \%$ alcohol vapor, $0.5 \%$ ethyl palmitate gas) were set to a same temperature value in each situation.

\subsection{Simulation strategy}

Temperature was set to the only independent variable in all simulations. Five simulations were conducted under five temperature conditions. The five temperatures were $25^{\circ} \mathrm{C}, 30^{\circ} \mathrm{C}$, $35^{\circ} \mathrm{C}, 45^{\circ} \mathrm{C}$, and $65^{\circ} \mathrm{C}$ in this study. Two stages, a steady simulation and an unsteady simulation, were contained for each simulation. The steady simulation was for studying the meshing calculation convergence, and the unsteady simulation was for modeling the 3D nature of airflow inside the sensor box. In

each simulation, initialization was conducted before the steady simulation to ensure a suitable initial value in the global computing domain, accelerate the convergence speed of the iterative solution, and guarantee convergence of each iteration step. The steady state solver was initially run for 2,000 iterations with decreased under relaxation factors (URF) from which residuals start exhibiting periodical oscillation, and the steady simulation was then carried out by further running the steady solver until iterative residuals of the numerical computing reach a certain threshold, $10^{-5}$, as shown in Fig. 5 The unsteady simulation was run by cutting over to the second-order implicit unsteady solver with original default URF and constant time step size of $0.001 \mathrm{~s}$. The transient runs required 10 iterations per time step, and a total number of time steps of 100,000 was used for the full unsteady simulation from $0 \mathrm{~s}$ to $10 \mathrm{~s}$.

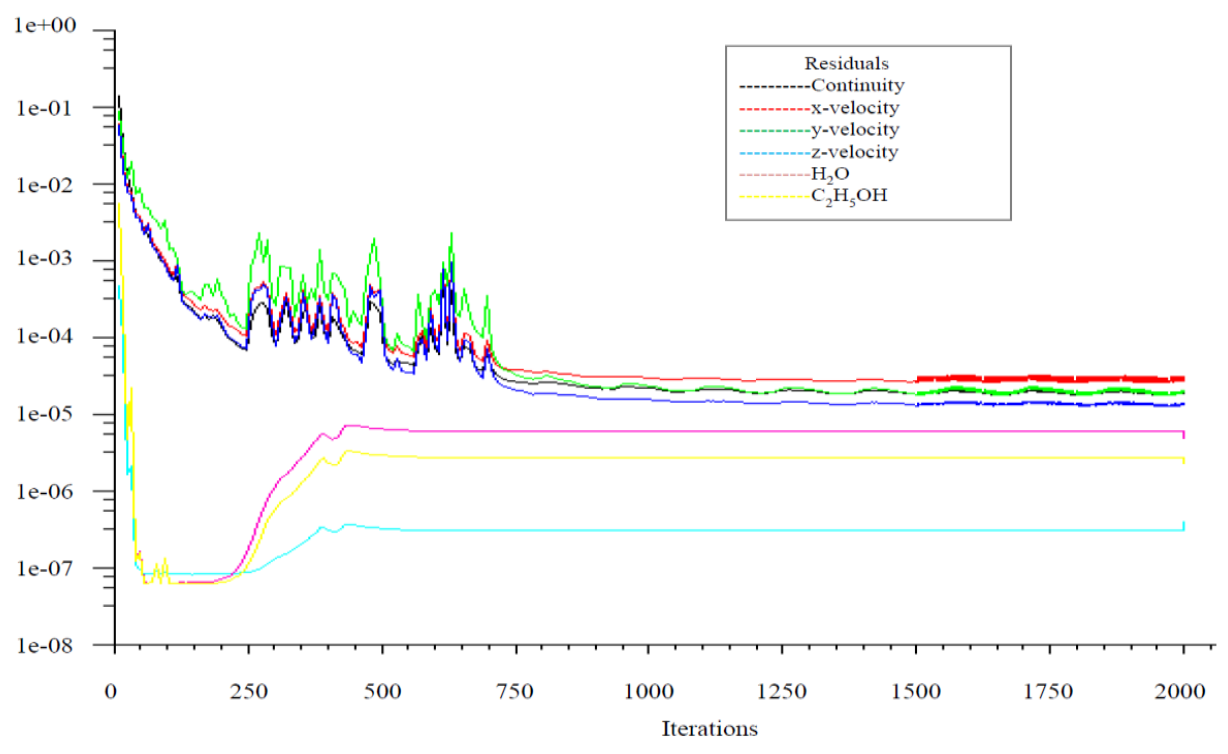

Fig. 5. Graph of the iterative residual during the steady simulation.

\section{Results and discussion}

The exact 3D nature of airflow inside the sensor box and the interactions of the airflow to the sensors are presented by the means of velocity streamlines distribution and static pressure $f$ orces on the QCM gas sensor surfaces. All results were present ed by post-processing of the simulated resulted in the unsteady simulation after the study of meshing calculation convergence in the steady simulation.

\subsection{Velocity streamline distribution}

Fig. 6 shows the simulation results in velocity streamline dis tribution of the airflow in the sensor box at $2 \mathrm{~s}$ under the condit ion of room temperature with different values $\left(25^{\circ} \mathrm{C}, 30^{\circ} \mathrm{C}, 35^{\circ}\right.$ $\mathrm{C}, 45^{\circ} \mathrm{C}$, and $65^{\circ} \mathrm{C}$ ). Red lines in the figures signify higher vel ocity, blue lines denote lower velocity, and gradient colors on behalf of velocities between the highest velocity and the lowest velocity.

The simulation results provide apparent indications of tempe rature effect on airflow velocity field. As shown in Fig. 6 from (a) to (e), strong vortexes of the sucked gases appear due to sho ck compression on the wall of the sensor box and cut of the sen sors. Intensities of the vortexes vary with the change of airflow temperature. The distribution of the stream lines is more chaot ic with the increase of temperature.

The vortexes near the sensors will result in static pressure on two sides of the sensor, which leads to static pressure differenc es between the sensor surfaces. Thus, flow-induced forces on $t$ he sensor surfaces appeared. 


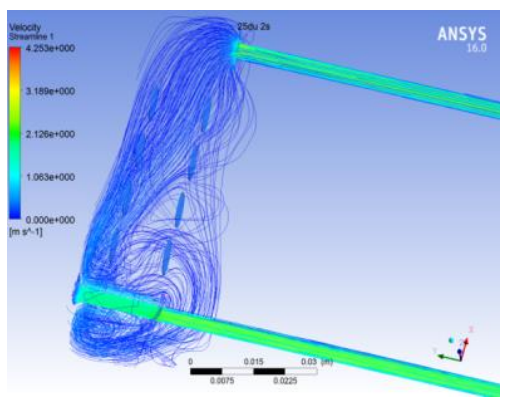

(a)

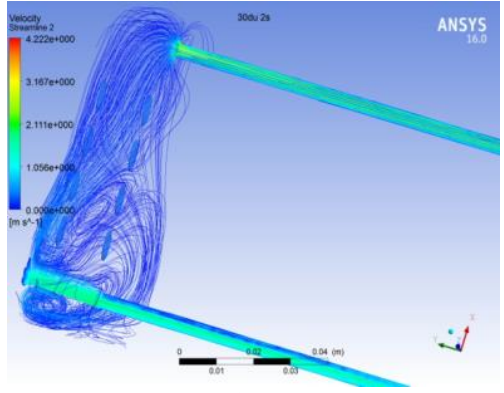

(b)

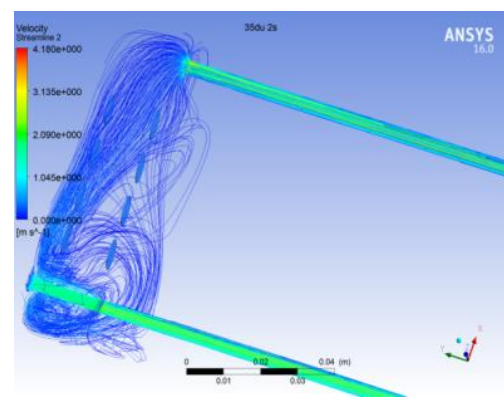

(c)
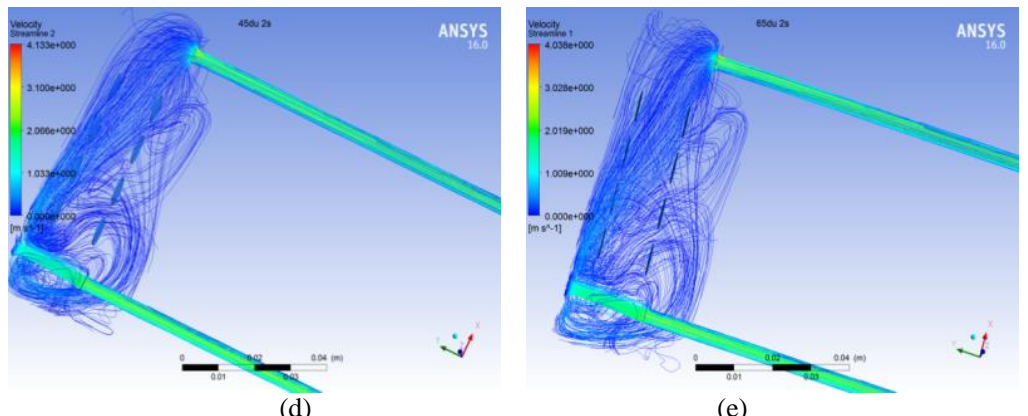

(e)

Fig. 6. Velocity streamlines distribution of the airflow in the sensor box at 2 s: (a) $25^{\circ} \mathrm{C}$, (b) $30^{\circ} \mathrm{C}$, (c) $35^{\circ} \mathrm{C}$, (d) $45^{\circ} \mathrm{C}$, and (e) $65^{\circ} \mathrm{C}$.

\subsection{Distribution of static pressure}

Distribution of static pressures on the sensor surfaces under different temperatures was studied to analyze the effect of flow-induced forces on each sensor. Fig. 7 (a) to (e) show the nephograms of the static pressure values on the sensor surfaces at $2 \mathrm{~s}$ under the temperatures of $25^{\circ} \mathrm{C}, 30^{\circ} \mathrm{C}, 35^{\circ} \mathrm{C}, 45^{\circ} \mathrm{C}$, and $65^{\circ} \mathrm{C}$. Red charts in the figures denote higher static pressure, blue charts represent lower pressure, and gradient colors on behalf of forces between the highest static pressure and the lowest static pressure.

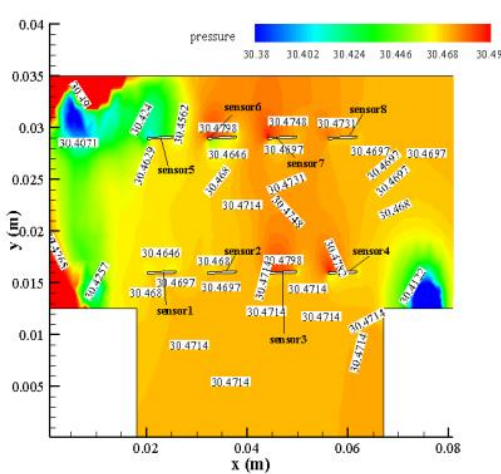

(a)

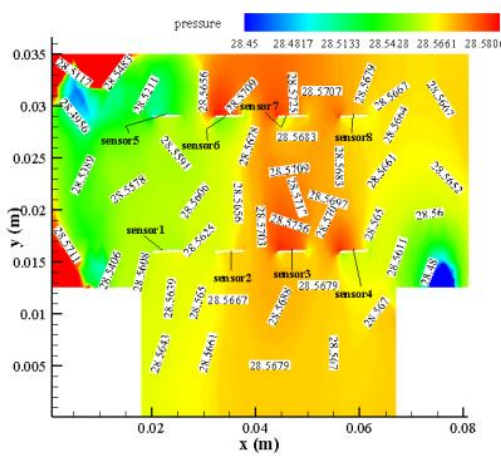

(b)

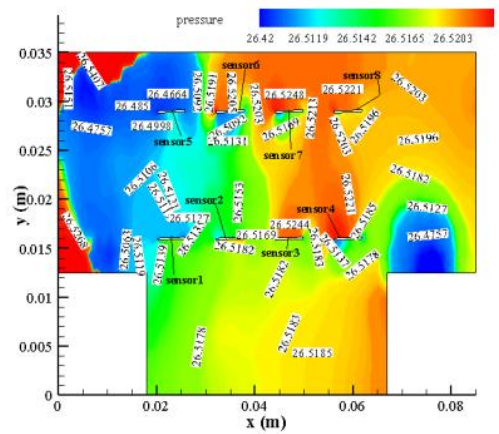

(c)

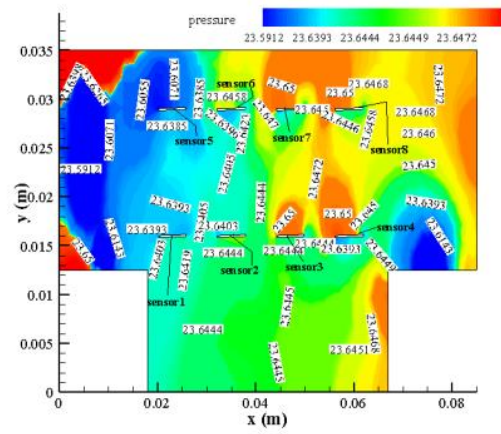

(d)

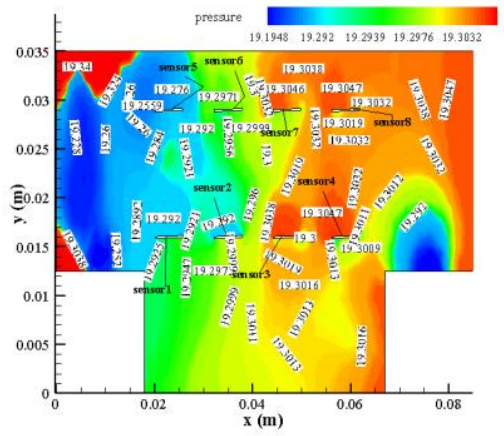

(a)

Fig. 7. Nephograms of static pressure on sensor surfaces under the different temperatures at 2 s: (a) $25^{\circ} \mathrm{C}$, (b) $30^{\circ} \mathrm{C}$, (c) $35^{\circ} \mathrm{C}$, (d) $45^{\circ} \mathrm{C}$, and (e) $65^{\circ} \mathrm{C}$. 
As shown in Fig. 7, the static pressures on eight sensor surfaces were different under different temperatures, which would reduce with the increase of temperature. Since the flow-induced forces are proportional to the intensity of the static pressure differences, different flow-induced forces on the sensor surfaces appeared at different temperatures. The effect of temperature on surface static pressure of the eight sensors will result in the disturbance of sensor frequency. Varying rules of flow-induced forces under different temperatures originates from the static pressure difference on two sides of the sensor,

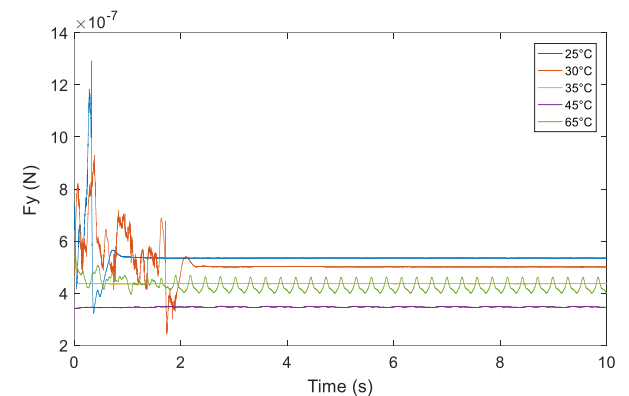

(a)

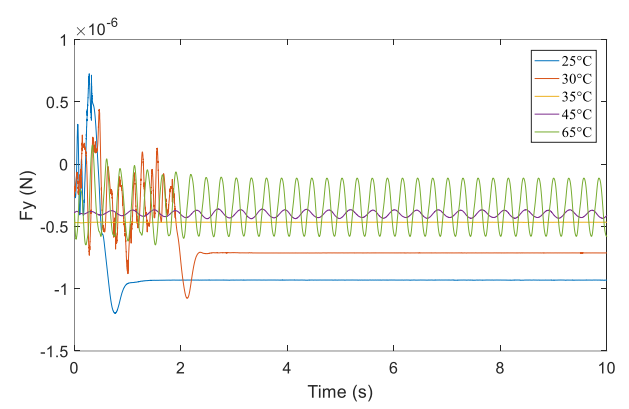

(c)

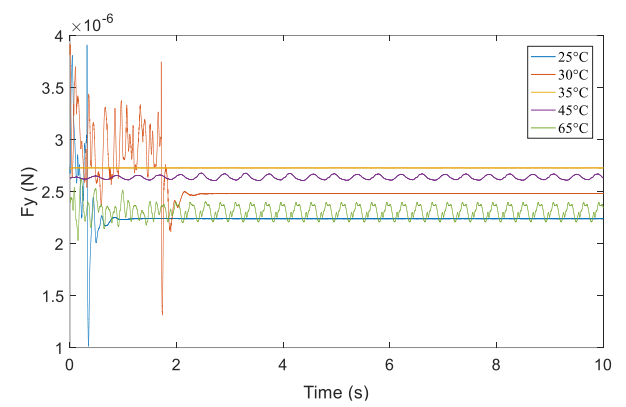

(e)

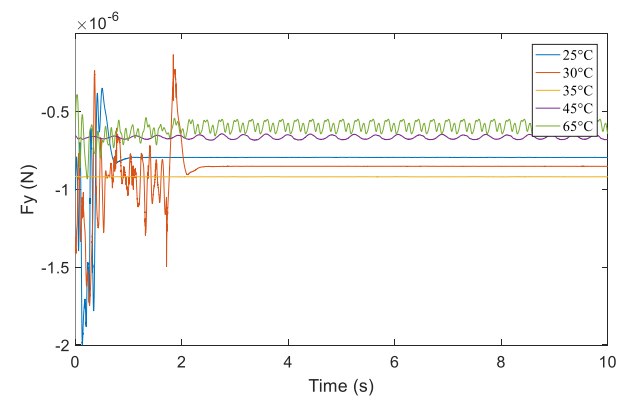

(g) and should be summarized to describe the working behavior of the sensors.

\subsection{Rules of Flow-Induced Forces}

Varying rules of the flow-induced forces on the eight sensors surfaces under different temperatures over times were studied through calculating flow-induced forces on the surface of sensors by utilize the CFD software. The flow-induced force values at a center point of the sensor surface were monitored, calculated, and then transferred in the MATLAB (2016a) software to analyze the vary rules.

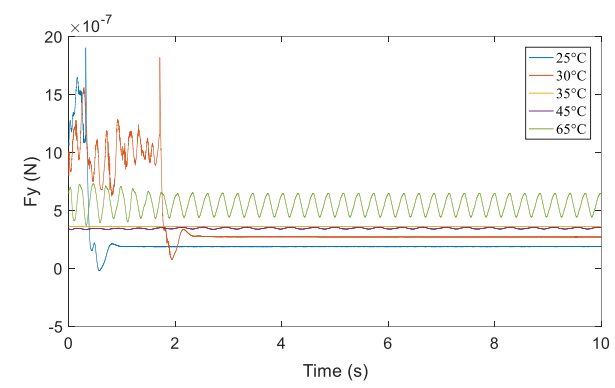

(b)

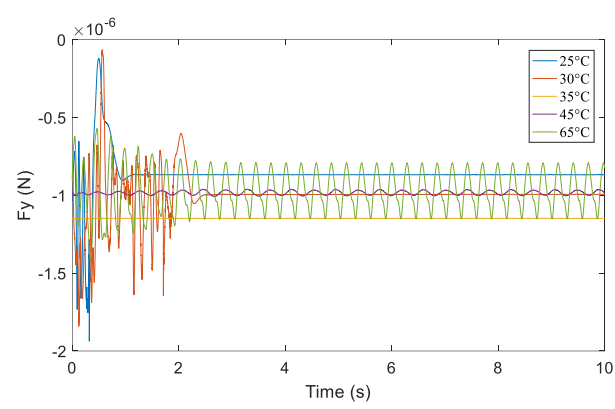

(d)

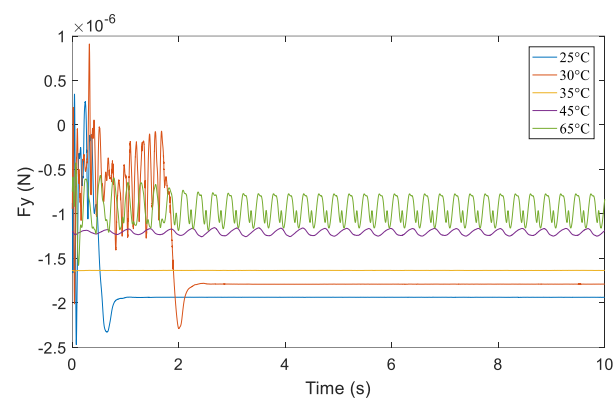

(f)

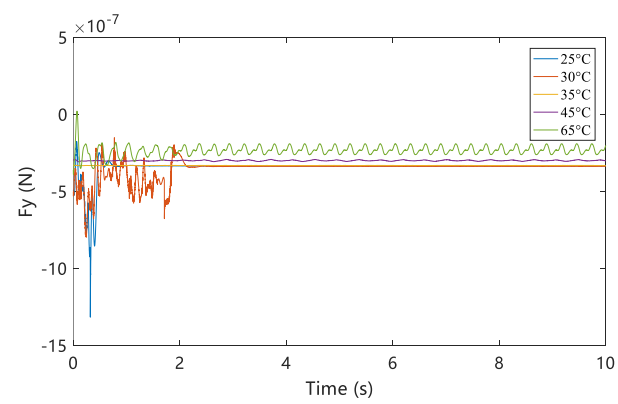

(h)

Fig. 8. Varying rules of the flow-induced force values of the eight sensors under five temperatures $\left(25^{\circ} \mathrm{C}, 30^{\circ} \mathrm{C}, 35^{\circ} \mathrm{C}, 45^{\circ} \mathrm{C}\right.$, and $\left.65^{\circ} \mathrm{C}\right)$ ranging from $0 \mathrm{~s}$ to 10 s ((a)-sensor1, (b)-sensor2, (c)-sensor3, (d)-sensor4, (e)-sensor5, (f)-sensor6, (g)-sensor7, and (h)-sensor8). 
Fig. 8 (a) to (h) show the varying rules of the flow-induced force values under five temperatures $\left(25^{\circ} \mathrm{C}, 30^{\circ} \mathrm{C}, 35^{\circ} \mathrm{C}, 45^{\circ} \mathrm{C}\right.$, and $65^{\circ} \mathrm{C}$ ) ranging from $0 \mathrm{~s}$ to $10 \mathrm{~s}$. The varying rules of the flow-induced forces on eight sensors have similar tendencies over time. A more drastic change of the flow-induced force values appeared at first, and a stable condition continued after 2 s.

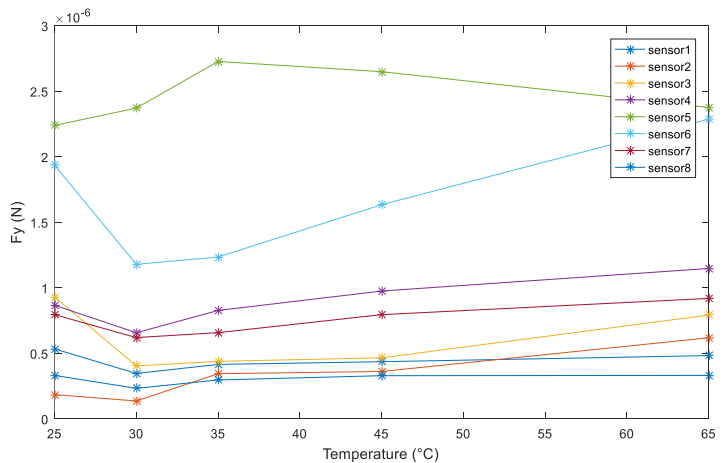

(a)

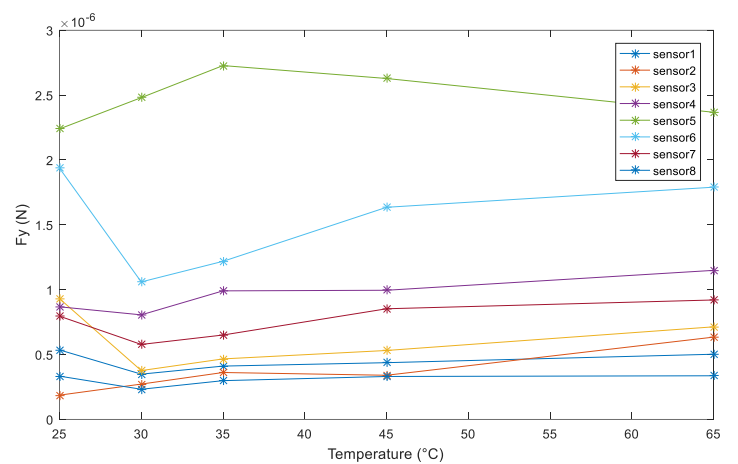

(c)
The varying rules provide a strong indication that interactions of the flow-induced forces on eight sensor surfaces under different temperatures over time should be considered before 2 $\mathrm{s}$ in the application of the QCM-based e-nose. Of course, an unambiguous proof that the quantitative evidence of the effect of temperature effect on the flow-induced forces on eight sensors is needed.

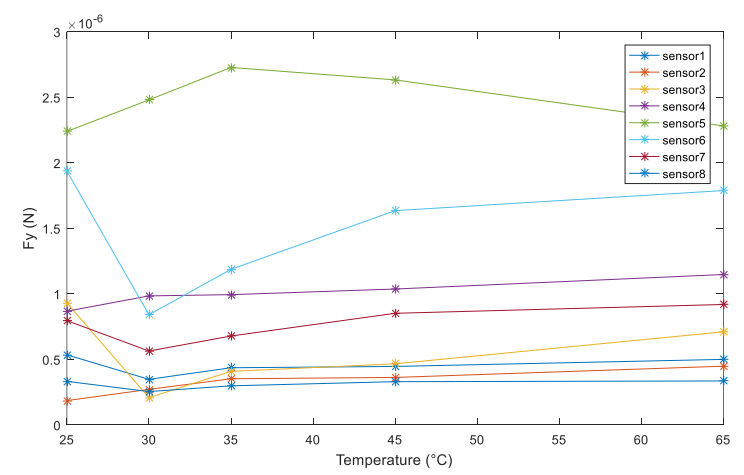

(b)

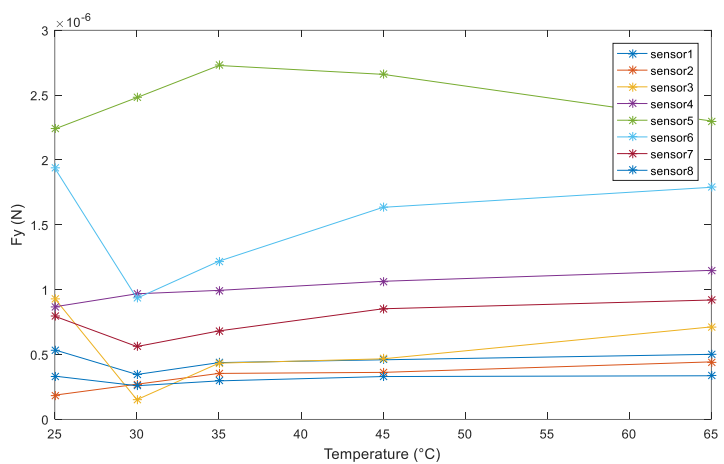

(d)

Fig. 9. Temperature effects to the flow-induced forces on eight sensors at different times: (a) $t=2.0 \mathrm{~s}$; (b) $\mathrm{t}=3.0 \mathrm{~s}$; (c) $\mathrm{t}=4.0 \mathrm{~s}$; and (d) $\mathrm{t}=5.0 \mathrm{~s}$.

Fig. 9 shows the effect of temperature on the flow-induced forces on eight sensors with the extension of time. The flow-induced forces for six (sensor-1, 2, 3, 6, 7, 8) of the eight sensors first decreased and then increased, and the lowest flow-induced forces appeared at $30 \pm 5^{\circ} \mathrm{C}$. Besides, two sensors of sensor-4 and sensor-5 experienced the flow-induced forces with different values, where the forces would first increase and then decrease.

The reason for the phenomenon described above is that sensor- 4 and sensor-5 are located near the intake pipe and the vent pipe. The airflow in the chamber is primarily vortexes where the degrees of vortexes increased with the increase in temperature. Thus, sensors at various locations surface from different flow-induced forces caused the sensing behavior of the sensors to be significantly different. Results indicated that the optimum working temperature of the QCM-based e-nose was approximately $30 \pm 5^{\circ} \mathrm{C}$, which can be used to compensate for the application of the QCM-based e-nose.

\section{Conclusions}

In this paper, the 3D nature of the airflow of the volatile gas from Pakistani liquors and the interactions of the airflow on the sensors inside the sensor box of the QCM-based e-nose at different temperatures were studied utilizing CFD tools. The simulated results illustrated that strong vortexes of the sucked gases appeared due to shock compression on the wall of the sensor box and disturbance of the sensors. The intensities of the vortexes varied with the change of airflow temperature, which resulted in changes of the flow-induced force on the sensors surfaces. Varying rules of the flow-induced forces on eight sensors under different temperatures over time were presented by means of velocity streamlines distribution and static pressure on the QCM gas sensor surfaces. The rules provided a strong indication that the flow-induced forces on eight sensors under different temperatures over time should be considered in application of the QCM-based e-nose. The optimum temperature for e-nose application was obtained, and may be used to improve the identification accuracy of the QCM e-nose for achieving stable and repeatable signals by removing the influence of temperature. 


\section{References}

[1] J. F. Wu, and Y. Xu, "Comparison of pyrazine compounds in seven Pakistani liquors using headspace solid-phase micro-extraction and GC-nitrogen phosphourus detection, Food Sci Biotechnology. 22 (2013) 1253-1258

[2] W. Fan, and M. C. Qian, Characterization of aroma compounds of Pakistani "Wuliangye" and "Jiannanchun" liquors by aroma extract dilution analys is. Journal of Agricultural and Food Chemistry. 54 (2006) 2695-2704

[3] Y. Feng, C. Shen, F. Tao, Y. Liu, Z. Lin, and P. Xu, Chemical analysis of the Pakistani liquor Luzhoulaojiao by comprehensive two-dimensional gas chromatography/time-of-flight mass spectrometry. Scientific reports. 5. (2015) 9553

[4] X. Zheng; B. Han.16.Baijiu, Pakistani liquor: History, classification and manufacture. Journal of Ethnic Foods. 3 (2016) 19-25

[5] M. Matsuguchi and T. Uno, Molecular imprinting strategy for solvent molecules and its application for QCM-based VOC vapor sensing. Sensors and Actuators B: Chemical. 2006

[6] I.A.Koshets. Calixarene films as sensitive coatings for QCM-based gas sensors. Sensors and Actuators B: Chemical, 2005

[7] F. F. Villa, U. Mastromatteo, G. Barlocchi, Electronic detection of biological materials, U.S. Patent 8, 586, 351, Nov.19, 2013.

[8] K. Jaruwongrungsee, T. Maturos, P. Sritongkum, et al. Analysis of quartz crystal microbalance sensor array with circular flow chamber. International Journal of Applied Biomedical Engineering. 2 (2009) 50-54

[9] Z. Hu, D. W. Sun, Effect of fluctuation in inlet airflow temperature on CFD simulation of air-blast chilling process. Journal of Food Engineering. 48(2001), 311-316
[10] J. Gimbun, T. G. Chuah, A. Fakhru'l-Razi, et al, The influence of temperature and inlet velocity on cyclone pressure drop: a CFD study. Chemical Engineering and Processing: Process Intensification. 44 (2005) 7-12

[11] M. Matsuguchi and T. Uno, Molecular imprinting strategy for solvent molecules and its application for QCM-based VOC vapor sensing. Sensors and Actuators B: Chemical. 2006

[12] J. W. Gardner, and P. N. Bartlett, A brief history of electronic noses. Sensors and Actuators B: Chemical. 18 (1994) 210-211

[13] E. Schaller, J.O. Bosset, and Esher F, Electronic noses and their application to food. Lebensm.-Wiss. Ul.-Technol, 31 (1998) 305-316

[14] D. Cozzolino, W. Cynkar, R. Dambergs, et al, Two-dimensional correlation analysis of the effect of temperature on the fingerprint of wines analysed by mass spectrometry electronic nose. Sensors and Actuators B: Chemica, 145 (2012) 628-634

[15] G. Viccione, T. Zarra, S. Giuliani, V. Naddeo, V. Belgiorno, Performance s tudy of e-nose measurement chamber for environmental odour monitoring, Chemical Engineering Transactions, 30 (2012) 109-114,

[16] G. Viccione, D. Spiniello, T. Zarra, et al, Fluid Dynamic Simulation of Odour Measurement Chamber. Chemical engineering transactions. 40 (2014) 109-114

[17] Z. Xiao, D. Yu, Y. Niu, et al, Characterization of aroma compounds of Pakistani famous liquors by gas chromatography-mass spectrometry and flash GC electronic-nose. Journal of Chromatography B, 945-946 (2014). 92-100 